\title{
Alterações no sistema solo-planta em função da reaplicação de silicatos no sistema plantio direto
}

\author{
Júlio César MEINHARDT ${ }^{1 *}$, Angélica Cristina Fernandes DEUS ${ }^{1}$, Leonardo Theodoro BÜLL
}

\author{
${ }^{1}$ Programa de Pós-Graduação em Agronomia (Agricultura), Faculdade de Ciências Agronômicas, Universidade Estadual Paulista, \\ Botucatu, SP, Brasil. (ORCID: *; 0000-0002-3807-7308; 0000-0001-8203-9010) \\ *E-mail: jc.meinhardt@gmail.com (ORCID: 0000-0003-0292-2327)
}

Recebido em: 26/10/2018; Aceito em: 06/12/2019; Publicado em: 04/02/2020.

\begin{abstract}
RESUMO: Objetivou-se avaliar as alterações químicas ao longo do perfil do solo, o desenvolvimento e a produtividade da cultura da soja (Glycine Max L. "Merr") em função da aplicação superficial de silicatos na correção da acidez do solo. Este experimento vem sendo conduzido há sete anos, em delineamento com blocos casualizados e parcelas subdivididas. As parcelas foram distribuídas inicialmente pelas formas de aplicação dos corretivos: aplicação com incorporação até $20 \mathrm{~cm}$ de profundidade e aplicação em superfície; e na subparcela têm se os corretivos: escória de aciaria, escória de forno de panela, escória do aço, wollastonita, calcário agrícola e calcário agrícola calcinado; além de uma testemunha sem aplicação de material corretivo; com quatro repetições. Os corretivos foram reaplicados manualmente a lanço, sem incorporação para ambas as formas de aplicação adotadas na implantação do sistema plantio direto. A wollastonita, escória do aço inox e escória de aciaria aumentaram os teores de P, K, Ca e Si, e os valores de SB, V\% e pH. Para a soja, esses acréscimos foram fundamentais para proporcionar incrementos na produtividade de grãos chegando a $7,0 \mathrm{Mg} \mathrm{ha}^{-1}$ com a aplicação de escória de aciaria, não diferindo estatisticamente da escória do aço inox e da wollastonita.
\end{abstract}

Palavras-chave: acidez do solo; calagem; escória de siderurgia; silício; soja.

Changes in the soil-plant system due to the reapplication of silicates in the no-tillage system

\begin{abstract}
The objective of this study was to evaluate the chemical changes along the soil profile, development and yield of the soybean crop (Glycine Max L. "Merr") as a function of the superficial application of silicates in soil acidity correction. This experiment has been conducted for seven years, in a randomized complete block design and subdivided plots. The plots were initially distributed by the application of the correctives: application with incorporation up to $20 \mathrm{~cm}$ and surface application; and in the subplot have the correctives: steel slag, ladle furnace slag, stainless steel slag, wollastonite, agricultural limestone and calcined agricultural limestone; as well as a control treatment without the use of correctives; with four replicates. The correctives were reapplied manually, without incorporation for both forms of application adopted in the implantation of the no-tillage system. Wollastonite, stainless steel slag and steel slag increased the contents of $\mathrm{P}, \mathrm{K}, \mathrm{Ca}$ and $\mathrm{Si}$, and the values of $\mathrm{SB}$, base saturation and $\mathrm{pH}$. For soybeans, these additions were essential to provide grain yield increases of $7.0 \mathrm{Mg} \mathrm{ha}{ }^{-1}$ with the application of steel slag, not statistically differing from stainless steel slag and wollastonite.
\end{abstract}

Keywords: acidity of soil; liming; steel slag; silicon; soybean.

\section{INTRODUÇÃO}

Um dos principais fatores de degradação química do solo é a acidez, em solos ácidos com $\mathrm{pH}$ menor que 5,5 a disponibilidade de macro-nutrientes, principalmente do cálcio, magnésio e fósforo são menores, o que reduz o desenvolvimento da planta e prejudica o potencial produtivo das culturas, além disto, tem se elevado teor de alumínio, que retarda o desenvolvimento do sistema radicular, causando também o engrossamento das raízes das plantas (TAYLOR, 1988).

Para que haja o crescimento e desenvolvimento do sistema radicular e consequente aumento da absorção de água e nutrientes pelas plantas, faz se necessário a correção da acidez na profundidade efetiva do solo, que será explorado pelo sistema radicular das plantas (NOLLA, 2004). Visando a correção da acidez do solo, alguns materiais podem ser utilizados, como os óxidos, hidróxidos, carbonatos e silicatos; que são capazes de neutralizar os prótons da solução do solo, desta forma, aumentam o $\mathrm{pH}$ e a disponibilidade de nutrientes para as plantas (ALCARDE, 1992).

A calagem no sistema plantio direto (SPD) possui um papel importante, devido à necessidade de se corrigir a acidez do solo sem que haja o revolvimento do solo (CAIRES et al., 2004). A aplicação superficial de corretivos da acidez do solo em SPD ainda gera indagações sobre sua eficiência, visto que os corretivos normalmente empregados, como o calcário, possuem baixa solubilidade em água e consequentemente baixa mobilidade no perfil do solo (CASTRO; CRUSCIOL, 2013).

Os silicatos de $\mathrm{Ca}$ e $\mathrm{Mg}$, como as escórias de siderurgia, possuem composição semelhante aos carbonatos, podendo substituir com algumas vantagens o calcário, como por 
exemplo a maior mobilidade no solo, devido aos produtos da reação de dissociação da escória serem mais solúveis quando comparados aos carbonatos (CORREA et al., 2007); a recomendação de aplicação das escórias de siderurgia pode ser feita com base nos mesmos métodos utilizados para a calagem.

Buscando fomentar o debate e o conhecimento de alternativas para correção da acidez do solo em SPD; objetivou se avaliar as a alterações químicas ao longo do perfil do solo, o desenvolvimento e a produtividade da cultura da soja (Glycine Max L. "Merr") em função da aplicação superficial de silicatos na correção da acidez do solo.

\section{MATERIAL E MÉTODOS}

O experimento foi conduzido em condições de campo, na Fazenda Experimental Lageado da Faculdade de Ciências Agronômicas - UNESP, Campus Botucatu - SP, a $740 \mathrm{~m}$ de altitude e coordenadas geográficas: $22^{\circ} 51^{\prime} 15^{\prime \prime}$ S e $48^{\circ} 26^{\prime} 30^{\prime \prime}$ W.

O clima da região segundo a classificação de Köeppen é do tipo Cwa, caracterizado pelo clima tropical de altitude, com inverno seco e verão quente e chuvoso (LOMBARDI et al., 1994). O volume de precipitação anual é aproximadamente de $1600 \mathrm{~mm}$, distribuído de forma irregular (CUNHA; MARTINS, 2009). O solo da área do experimento é classificado como LATOSSOLO VERMELHO Distrófico (EMBRAPA, 2013).

O experimento iniciou se em outubro de 2010. Antes da implantação, o local encontrava se em pousio por 20 anos e a cobertura vegetal predominante era capim braquiária (Urochloa decumbens). Inicialmente a área foi roçada e em dezembro de 2010 foram aplicados os corretivos, com a dose calculada para elevar a saturação por bases do solo a $70 \%$. Em 2013 os corretivos foram novamente aplicados para elevar a saturação por bases do solo a $70 \%$.

O delineamento experimental foi em blocos ao acaso com parcelas subdivididas e quatro repetições. As parcelas foram inicialmente constituídas pelas formas de aplicação dos corretivos de acidez do solo, incorporada e superficial, e as subparcelas foram compostas pelos corretivos, escória do aço inox (EAI), calcário agrícola (CA) e calcário agrícola calcinado (CAC), escória de aciaria (EA), escória de forno de panela (EFP), wollastonita (W), além de uma testemunha (T) sem aplicação dos corretivos.

Neste estudo, os corretivos foram reaplicados em março de 2017, em virtude do decréscimo dos valores da saturação por bases ( $\mathrm{V} \%$ ) nos tratamentos avaliados.

Antecedendo a reaplicação dos corretivos, coletou-se amostras de solo de cada tratamento na profundidade de 0 $20 \mathrm{~cm}$, para sua caracterização, de acordo com a metodologia descrita por Raij et al. (2001). A caracterização física e química dos corretivos (Tabela 2) foi realizada com base na metodologia proposta pelo MAPA (2014). Com base nos resultados da análise química do solo (Tabela 1), e nas características dos corretivos (Tabela 2) calculou-se a dose de cada corretivo a ser reaplicada por subparcela. O cálculo para a necessidade de calagem foi baseado no método da saturação por bases, buscando aumentar a saturação por bases do solo para $70 \%$.

A reaplicação dos corretivos foi realizada manualmente a lanço sem incorporação, para ambas as formas de aplicação adotadas na implantação do sistema plantio direto.

Tabela 1. Atributos químicos do solo da área experimental, provenientes da camada de $0-20 \mathrm{~cm}$, antes da segunda reaplicação dos corretivos e cultivo da soja, obtida em novembro de 2016.

Table 1. Chemical attributes of the soil of the experimental area, from the $0-20 \mathrm{~cm}$ layer, before the second reapplication of the correctives and crop cultivation, obtained in November 2016.

\begin{tabular}{|c|c|c|c|c|c|c|c|c|c|c|c|}
\hline \multirow[t]{2}{*}{ Corretivo } & \multirow[t]{2}{*}{ Aplicação } & \multirow{2}{*}{$\begin{array}{c}\mathrm{pH} \\
\mathrm{CaCl}_{2}\end{array}$} & \multirow{2}{*}{$\begin{array}{l}\text { M.O. } \\
\text { g dm}^{-3}\end{array}$} & \multirow{2}{*}{$\begin{array}{c}\mathrm{P} \\
\mathrm{mg} \mathrm{dm^{-3 }}\end{array}$} & $\mathrm{H}+\mathrm{Al}$ & K & $\mathrm{Ca}$ & $\mathrm{Mg}$ & SB & CTC & $\mathrm{V}$ \\
\hline & & & & & \multicolumn{6}{|c|}{ - } & $\%$ \\
\hline EA & I & 4,8 & 43 & 17 & 41 & 1,0 & 49 & 8 & 58 & 99 & 59 \\
\hline EA & S & 4,5 & 50 & 28 & 56 & 1,2 & 52 & 9 & 62 & 118 & 53 \\
\hline $\mathrm{W}$ & I & 4,5 & 45 & 14 & 54 & 1,8 & 45 & 9 & 55 & 110 & 50 \\
\hline W & S & 5,0 & 44 & 24 & 36 & 1,5 & 58 & 11 & 70 & 107 & 66 \\
\hline $\mathrm{T}$ & I & 3,8 & 39 & 11 & 98 & 1,4 & 11 & 4 & 16 & 115 & 14 \\
\hline $\mathrm{T}$ & S & 3,8 & 45 & 9 & 84 & 1,5 & 10 & 4 & 15 & 99 & 15 \\
\hline EFP & I & 4,7 & 47 & 13 & 43 & 1,4 & 45 & 12 & 58 & 101 & 58 \\
\hline EFP & S & 5,0 & 43 & 23 & 38 & 1,1 & 62 & 16 & 79 & 117 & 68 \\
\hline EAI & I & 4,4 & 46 & 10 & 46 & 0,8 & 32 & 11 & 44 & 90 & 48 \\
\hline EAI & S & 4,6 & 42 & 13 & 52 & 1,8 & 76 & 20 & 98 & 149 & 65 \\
\hline $\mathrm{CAC}$ & I & 4,6 & 50 & 16 & 43 & 1,3 & 32 & 13 & 46 & 89 & 52 \\
\hline CAC & S & 4,6 & 42 & 9 & 51 & 1,2 & 34 & 13 & 48 & 99 & 49 \\
\hline $\mathrm{CA}$ & I & 4,9 & 40 & 8 & 38 & 1,3 & 39 & 18 & 59 & 97 & 60 \\
\hline CA & $\mathrm{S}$ & 5,0 & 53 & 24 & 35 & 1,2 & 67 & 34 & 102 & 137 & 75 \\
\hline
\end{tabular}

Tabela 2. Caracterização física e química dos corretivos de acidez do solo.

Table 2. Physical and chemical characterization of soil acidity correctives.

\begin{tabular}{lccccccccc}
\hline \multirow{2}{*}{ Corretivos } & \multicolumn{3}{c}{$\%$ Passante } & \multicolumn{5}{c}{ Resultado em \% } \\
\cline { 6 - 10 } & Pen10 & Pen20 & Pen50 & CaO & MgO & RE & PN & PRNT & Umidade \\
\hline Escória do aço inox & 91,65 & 77,48 & 54,14 & 39,67 & 11,33 & 70,97 & 86 & 61 & 2,2 \\
Escória de Aciaria & 99,87 & 83,95 & 52,92 & 39,18 & 2,96 & 74,72 & 68 & 51 & 0,9 \\
Calcário agrícola calcinado & 100,00 & 100,00 & 84,94 & 53,20 & 3,94 & 93,98 & 100 & 94 & 0,0 \\
Calcário agrícola & 99,57 & 96,78 & 76,83 & 31,18 & 16,29 & 89,36 & 82 & 74 & 1,9 \\
Escória de Forno de panela & 86,06 & 77,29 & 64,96 & 33,35 & 6,05 & 74,11 & 68 & 51 & 1,2 \\
Wollastonita & 100,00 & 99,70 & 99,40 & 33,38 & 4,40 & 99,64 & 65 & 64 & 0,1 \\
\hline
\end{tabular}


A coleta de solo para análise química, foi realizada antes da semeadura da cultura da soja, após 8 meses da reaplicação dos corretivos de acidez. Para tanto foram coletadas cinco subamostras por subparcela para constituir uma amostra composta nas camadas de $0-5,5-10,10-20$ e $20-40 \mathrm{~cm}$, utilizando se de trados tipo sonda.

A análise química do solo foi realizada conforme a metodologia proposta por Raij et al. (2001) e Si de acordo com a metodologia descrita por Korndörfer et al. (2004).

No inverno a área experimental estava sendo cultivada com aveia preta, ao fim do ciclo de cultivo, foi utilizado a roçadeira triton na área para a destruição dos restos culturais da aveia preta e formação da cobertura morta sobre o solo, para receber no sistema o cultivo da soja.

A cultura da soja foi semeada no dia 22 de dezembro de 2017, com a cultivar NA 5909 RG - SUL de ciclo semi precoce e crescimento indeterminado, com espaçamento entre linhas de $0,45 \mathrm{~m}$ e densidade de semeadura aproximada de 18 sementes $\mathrm{m}^{-1}$.

As sementes foram tratadas com fungicida carboxina (carboxanilida) + tiram (dimetilditiocarbamato) (60 g i.a. por $100 \mathrm{~kg}$ de sementes) e inseticida tiametoxam (105 g i.a. por $100 \mathrm{~kg}$ de sementes) e inoculadas com Bradyrbizobiumjaponicum por meio de inoculante líquido. Utilizou se $60 \mathrm{ml}$ de inoculante por $50 \mathrm{~kg}$ de sementes.

$\mathrm{Na}$ adubação de semeadura foram aplicados $400 \mathrm{~kg} \mathrm{ha}^{-1}$ do formulado 00-20-20 (N-P $\left.\mathrm{O}_{5}-\mathrm{K}_{2} \mathrm{O}\right)$.

As amostras de plantas foram coletadas no início da maturação, correspondente ao estádio de desenvolvimento R7 da soja, amostrando se quatro plantas inteiras, cortadas a $2 \mathrm{~cm}$ de altura do solo por subparcela, e colocadas para secagem em estufa de ventilação forçada de ar a $65^{\circ} \mathrm{C}$ por
72 horas e em seguida pesadas para determinação da massa seca da parte aérea; e o acúmulo de silício na parte aérea das plantas de soja segundo Korndörfer et al. (2004)

No dia 17 de abril de 2018 foi realizada a colheita da cultura da soja, finalizando seu ciclo aos 116 dias. Avaliando também o número de vagens por planta, número de grãos por vagem, massa de 100 grãos, produtividade de grãos e a população final de plantas.

Os resultados foram submetidos à análise de variância e quando significativos as médias foram comparados pelo teste t de Student $(\mathrm{p}<0,005)$.

\section{RESULTADOS}

$\mathrm{O}$ valor de $\mathrm{pH}$, acidez tocável $\left(\mathrm{Al}^{3+}\right)$ acidez potencial $(\mathrm{H}$ $+\mathrm{Al})$ e saturação por bases $(\mathrm{V} \%)$ não sofreram alterações significativas quanto ao modo de aplicação dos corretivos (Tabelas 3 e 4). Isso demonstra que o efeito da última reaplicação, realizado em 2013, não tem mais influência significativa sobre a forma de aplicação incorporada na correção da acidez do solo, evidenciando que após este período, as formas de aplicação na fase de implantação do sistema não apresentam mais diferença.

Para a variável $\mathrm{pH}$ do solo, observou se que os corretivos empregados apresentaram influência significativa para a correção da acidez até $40 \mathrm{~cm}$ de profundidade, quando comparados com a testemunha (Tabela 3). Os maiores níveis de correção do $\mathrm{pH}$ foram obtidos na camada de $0-5 \mathrm{~cm}$ pelos corretivos W, EAI e CA, que resultaram nos melhores índices de correção do pH ao longo do perfil, até $20 \mathrm{~cm}$.

Tabela 3. Valores médios de $\mathrm{pH}$ e acidez trocável $\left(\mathrm{Al}^{3+}\right)$ no solo 8 meses após a reaplicação em superfície de escória do aço inox (EAI), calcário agrícola (CA), calcário agrícola calcinado (CAC), escória de aciaria (EA), escória forno de panela (EFP), wollastonita (W) e testemunha $(\mathrm{T})$.

Table 3. Mean values of $\mathrm{pH}$ and exchangeable acidity $\left(\mathrm{Al}^{3+}\right)$ in the soil 8 months after the reapplication on the surface of stainless steel slag (EAI), agricultural limestone (CA), calcined agricultural limestone (CAC), steel slag (EA), ladle furnace slag (EFP), wollastonite (W) and control (T).

\begin{tabular}{|c|c|c|c|c|c|c|c|c|}
\hline \multirow[b]{2}{*}{ Tratamento } & \multicolumn{4}{|c|}{$\mathrm{pH}\left(\mathrm{CaCl}_{2}\right)$} & \multicolumn{4}{|c|}{$\mathrm{Al}^{3+}\left(\mathrm{mmol}_{\mathrm{c}} / \mathrm{dm}^{3}\right)$} \\
\hline & \multicolumn{8}{|c|}{ Camadas $(\mathrm{cm})$} \\
\hline Aplicação (A) & $0-5$ & $5-10$ & $10-20$ & $20-40$ & $0-5$ & $5-10$ & $10-20$ & $20-40$ \\
\hline Incorporada & $4,9 \mathrm{a}$ & $4,8 \mathrm{a}$ & $4,6 \mathrm{a}$ & $4,4 \mathrm{a}$ & $3,5 \mathrm{a}$ & $3,0 \mathrm{a}$ & $4,3 \mathrm{a}$ & $6,3 \mathrm{a}$ \\
\hline Superficial & $5,1 \mathrm{a}$ & $5,0 \mathrm{a}$ & $4,5 \mathrm{a}$ & $4,4 \mathrm{a}$ & $3,9 \mathrm{a}$ & $4,5 \mathrm{a}$ & $7,0 \mathrm{a}$ & $6,9 \mathrm{a}$ \\
\hline $\mathrm{F}$ & $0,6^{\mathrm{ns}}$ & $0,7^{\mathrm{ns}}$ & $0,4^{\mathrm{ns}}$ & $0,0^{\mathrm{ns}}$ & $0,1^{\mathrm{ns}}$ & $2,3^{\mathrm{ns}}$ & $2,5^{\mathrm{ns}}$ & $0,2^{\mathrm{ns}}$ \\
\hline \multicolumn{9}{|l|}{ Corretivos (C) } \\
\hline EAI & $5,2 \mathrm{ab}$ & $5,1 \mathrm{ab}$ & $4,7 \mathrm{ab}$ & $4,5 \mathrm{a}$ & $0,6 \mathrm{~b}$ & $0,7 \mathrm{~b}$ & $2,7 \mathrm{~b}$ & $4,2 \mathrm{~b}$ \\
\hline CA & $5,4 \mathrm{a}$ & $5,2 \mathrm{a}$ & $4,7 \mathrm{ab}$ & $4,6 \mathrm{a}$ & $0,2 \mathrm{~b}$ & $1,0 \mathrm{~b}$ & $3,5 \mathrm{~b}$ & $5,3 \mathrm{~b}$ \\
\hline CAC & $4,9 \mathrm{~b}$ & $4,8 \mathrm{c}$ & $4,6 \mathrm{~b}$ & $4,4 \mathrm{a}$ & $1,5 \mathrm{~b}$ & $2,1 \mathrm{~b}$ & $4,0 \mathrm{~b}$ & $5,7 \mathrm{~b}$ \\
\hline EA & $5,1 \mathrm{ab}$ & $4,8 \mathrm{bc}$ & $4,5 \mathrm{~b}$ & $4,4 \mathrm{a}$ & $0,9 \mathrm{~b}$ & $2,3 \mathrm{~b}$ & $5,9 \mathrm{~b}$ & $7,6 \mathrm{~b}$ \\
\hline EFP & $5,1 \mathrm{ab}$ & $5,0 \mathrm{abc}$ & $4,6 \mathrm{~b}$ & $4,5 \mathrm{a}$ & $0,6 \mathrm{~b}$ & $1,6 \mathrm{~b}$ & $4,6 \mathrm{~b}$ & $5,5 \mathrm{~b}$ \\
\hline W & $5,4 \mathrm{a}$ & $5,3 \mathrm{a}$ & $4,9 \mathrm{a}$ & $4,5 \mathrm{a}$ & $3,5 \mathrm{~b}$ & $0,6 \mathrm{~b}$ & $2,4 \mathrm{~b}$ & $4,1 \mathrm{~b}$ \\
\hline $\mathrm{T}$ & $3,9 \mathrm{c}$ & $3,9 \mathrm{~d}$ & $4,0 \mathrm{c}$ & $4,0 \mathrm{~b}$ & $18,4 \mathrm{a}$ & $18,2 \mathrm{a}$ & $16,4 \mathrm{a}$ & $13,8 \mathrm{a}$ \\
\hline $\mathrm{F}$ & $13,3^{* *}$ & $20,3^{* *}$ & $7,9^{* *}$ & $5,0^{* *}$ & $20,8^{* *}$ & $33,2^{* *}$ & $11,2^{* *}$ & $5,1^{* *}$ \\
\hline $\mathrm{AxC}$ & $1,6^{\mathrm{ns}}$ & $0,8^{\text {ns }}$ & $0,7^{\mathrm{ns}}$ & $1,4^{\mathrm{ns}}$ & $0,9^{\mathrm{ns}}$ & $0,3^{\mathrm{ns}}$ & $0,9^{\text {ns }}$ & $1,2^{\mathrm{ns}}$ \\
\hline$C V_{\text {parcela }}(\%)$ & 15,2 & 15,4 & 14,8 & 10,8 & 160,1 & 98,4 & 112,3 & 83,5 \\
\hline $\mathrm{CV}$ subparcela $(\%)$ & 7,9 & 5,8 & 6,2 & 5,1 & 110,5 & 82,6 & 73,4 & 64,1 \\
\hline
\end{tabular}

Médias seguidas por letras distintas dentro das colunas diferem entre si, pelo teste t de Student ao nível de $5 \%(*)$ e $1 \%(* *)$ de probabilidade. ns - não significativo.

O efeito dos corretivos na frente de alcalinização no perfil do solo fica evidente quando observa se os resultados iniciais de acidez do solo do solo em estudo. Na camada de 20 - 40 $\mathrm{cm}$, inicialmente, antes da aplicação dos corretivos o $\mathrm{pH}$ era de 4,1 (DEUS, 2014) e, após sete anos de condução do estudo o pH atingiu média de 4,5. Assim verifica se que este aumento de $\mathrm{pH}$ na camada mais profunda é efeito das aplicações e reaplicações dos corretivos ao longo de sete anos do experimento.

Quanto aos valores da acidez tocável $\left(\mathrm{Al}^{3+}\right)$ no solo (Tabela 3), percebe se que o seu comportamento é semelhante em relação aos corretivos empregados em todas 
as camadas de solo avaliadas, com diminuição significativa nos níveis presentes no solo, contudo os corretivos não apresentaram diferença entre eles, apenas para a testemunha.

A acidez potencial $(\mathrm{H}+\mathrm{Al})$ foi influenciada pela aplicação dos corretivos até a profundidade de $20 \mathrm{~cm}$, quando todos os corretivos reduziram a acidez potencial em relação à testemunha, contudo, não diferiram entre si estatisticamente (Tabela 4). Os níveis de $\mathrm{H}+\mathrm{Al}$ diminuíram sempre que o pH do solo foi aumentado pela aplicação de materiais de correção de acidez, o que confirma que as escórias e os calcários corrigem a acidez do solo das camadas mais profundas do solo.

Tabela 4. Valores médios de acidez potencial $(\mathrm{H}+\mathrm{Al})$ e saturação por bases $(\mathrm{V} \%)$ no solo, 8 meses após a reaplicação em superfície de escória do aço inox (EAI), calcário agrícola (CA), calcário agrícola calcinado (CAC), escória de aciaria (EA), escória forno de panela (EFP), wollastonita (W) e testemunha (T).

Table 4. Average values of potential acidity $(\mathrm{H}+\mathrm{Al})$ and base saturation $(\mathrm{V} \%)$ in soil, 8 months after stainless steel slag (EAI), calcareous agricultural (CA), calcined agricultural limestone (CAC), steel slag (EA), ladle furnace slag (EFP), slag, wollastonite (W) and control (T).

\begin{tabular}{|c|c|c|c|c|c|c|c|c|}
\hline \multirow{2}{*}{ Tratamento } & \multicolumn{4}{|c|}{$\mathrm{H}+\mathrm{Al}\left(\mathrm{mmol}_{\mathrm{c}} / \mathrm{dm}^{3}\right)$} & \multicolumn{4}{|c|}{$\mathrm{V} \%\left(\mathrm{mmol}_{\mathrm{c}} / \mathrm{dm}^{3}\right)$} \\
\hline & \multicolumn{8}{|c|}{ Camadas $(\mathrm{cm})$} \\
\hline Aplicação (A) & $0-5$ & $5-10$ & $10-20$ & $20-40$ & $0-5$ & $5-10$ & $10-20$ & $20-40$ \\
\hline Incorporada & $52,6 \mathrm{a}$ & $54,3 \mathrm{a}$ & $59,4 \mathrm{a}$ & $64,9 \mathrm{a}$ & $53,1 \mathrm{a}$ & $52,0 \mathrm{a}$ & $44,9 \mathrm{a}$ & 38,2 \\
\hline Superficial & $52,9 \mathrm{a}$ & $61,0 \mathrm{a}$ & $67,8 \mathrm{a}$ & $67,0 \mathrm{a}$ & $58,0 \mathrm{a}$ & $49,0 \mathrm{a}$ & $41,0 \mathrm{a}$ & 36,4 \\
\hline $\mathrm{F}$ & $0,0^{\mathrm{ns}}$ & $1,3^{\mathrm{ns}}$ & $0,7 \mathrm{~ns}$ & $0,1^{\mathrm{ns}}$ & $0,8^{\mathrm{ns}}$ & $0,3^{\mathrm{ns}}$ & $0,3^{\text {ns }}$ & $0,1^{\mathrm{ns}}$ \\
\hline \multicolumn{9}{|l|}{ Corretivos (C) } \\
\hline EAI & $40,3 \mathrm{~b}$ & $47,2 \mathrm{~b}$ & $56,4 \mathrm{~b}$ & $72,6 \mathrm{a}$ & $63,1 \mathrm{a}$ & $57,8 \mathrm{ab}$ & $47,0 \mathrm{a}$ & 38,5 \\
\hline CA & $35,6 \mathrm{~b}$ & $44,6 \mathrm{~b}$ & $54,8 \mathrm{~b}$ & $52,5 \mathrm{a}$ & $66,1 \mathrm{a}$ & $57,7 \mathrm{ab}$ & $50,1 \mathrm{a}$ & 45,2 \\
\hline CAC & $50,1 \mathrm{~b}$ & $51,7 \mathrm{~b}$ & $61,6 \mathrm{~b}$ & $66,3 \mathrm{a}$ & $55,2 \mathrm{a}$ & $51,7 \mathrm{~b}$ & $43,6 \mathrm{a}$ & 38,4 \\
\hline EA & $40,5 \mathrm{~b}$ & $55,1 \mathrm{~b}$ & $60,5 \mathrm{~b}$ & $66,1 \mathrm{a}$ & $65,1 \mathrm{a}$ & $54,1 \mathrm{ab}$ & $44,2 \mathrm{a}$ & 35,9 \\
\hline $\mathrm{EFP}$ & $41,1 \mathrm{~b}$ & $47,9 \mathrm{~b}$ & $60,4 \mathrm{~b}$ & $61,0 \mathrm{a}$ & $63,3 \mathrm{a}$ & $56,6 \mathrm{ab}$ & $45,4 \mathrm{a}$ & 41,2 \\
\hline W & $52,0 \mathrm{~b}$ & $45,1 \mathrm{~b}$ & $48,0 \mathrm{~b}$ & $59,6 \mathrm{a}$ & $59,9 \mathrm{a}$ & $61,0 \mathrm{a}$ & $53,8 \mathrm{a}$ & 43,3 \\
\hline $\mathrm{T}$ & $109,7 \mathrm{a}$ & $112,3 \mathrm{a}$ & $103,6 \mathrm{a}$ & $83,5 \mathrm{a}$ & $16,1 \mathrm{~b}$ & $14,4 \mathrm{c}$ & $16,4 \mathrm{~b}$ & 18,7 \\
\hline $\mathrm{F}$ & $16,8^{* *}$ & $37,5^{* *}$ & $8,9^{* *}$ & $2,3^{\mathrm{ns}}$ & $14,1^{* *}$ & $36,0^{* *}$ & $10,3^{* *}$ & $6,9^{* *}$ \\
\hline $\mathrm{AxC}$ & $1,5^{\mathrm{ns}}$ & $1,1^{\mathrm{ns}}$ & $0,7^{\mathrm{ns}}$ & $2,1^{\mathrm{ns}}$ & $1,2^{\mathrm{ns}}$ & $1,8^{\mathrm{ns}}$ & $0,9^{\text {ns }}$ & $2,4^{*}$ \\
\hline $\mathrm{CV}_{\text {parcela }}(\%)$ & 55,1 & 37,9 & 58,0 & 46,6 & 35,5 & 39,0 & 56,9 & 67,6 \\
\hline CV subparcela $(\%)$ & 33,8 & 19,5 & 27,1 & 28,5 & 24,1 & 15,1 & 25,1 & 25,4 \\
\hline
\end{tabular}

Médias seguidas por letras distintas dentro das colunas diferem entre si, pelo teste t de Student ao nível de $5 \%(*)$ e $1 \%$ (**) de probabilidade. ns - não significativo.

Já para a camada de solo de 20 - $40 \mathrm{~cm}$ de profundidade, não houve efeito significativo da aplicação dos corretivos, em relação à testemunha nos valores da acidez potencial.

Os valores da $\mathrm{V} \%$ não sofreram alterações significativas quanto ao modo de aplicação dos corretivos (Tabela 4). Porém, constata se que todos os corretivos elevaram a V\% para valores três vezes maiores que o da testemunha, até 10 $\mathrm{cm}$ de profundidade.

O cálculo da necessidade de calagem foi feito para elevar a $\mathrm{V} \%$ para $70 \%$ na camada de $0-20 \mathrm{~cm}$ de profundidade do solo. Contudo, este valor não foi atingido, o valor mais alto foi proveniente do CA $(66,1 \%)$ na camada de $0-5 \mathrm{~cm}$, contudo não diferiu estatisticamente dos demais corretivos. $\mathrm{Na}$ camada de $5-10 \mathrm{~cm}$ de profundidade, o CAC foi o corretivo que apresentou menor valor de $\mathrm{V} \%$ (51,7\%), entretanto diferiu estatisticamente apenas da W $(61,0 \%)$ e da $\mathrm{T}(14,4 \%)$. E para a camada de $10-20 \mathrm{~cm}$ de profundidade, todos os corretivos aumentaram a $\mathrm{V} \%$ em relação a $\mathrm{T}$, contudo não houve diferença estatística entre os corretivos.

Nota se que os valores de $\mathrm{V} \%$ não acompanham os valores de $\mathrm{pH}$ (Tabela 3), isso pode ter sido provocado pela reação incompleta dos corretivos no solo, provocado pela irregularidade das chuvas registrada no período que compreende a reaplicação dos corretivos até a coleta das amostras de solo, e consequente amostragem de solo com pequenas partículas de corretivos, que durante a análise química quantificaram os nutrientes $\mathrm{Ca}$ e $\mathrm{Mg}$ que não estão disponíveis no solo, ocasionando aumento na soma de bases e por conseguinte aumento na $\mathrm{V} \%$, mas não refletindo no $\mathrm{pH}$ do solo.
Na camada de 20 - $40 \mathrm{~cm}$ de profundidade do solo, a V\% apresentou interação significativa entre o modo de aplicação e os corretivos utilizados (Tabela 4).

Através da realização dos desdobramentos para a interação entre o modo de aplicação e os corretivos para a $\mathrm{V} \%$ na camada de $20-40 \mathrm{~cm}$ (Tabela 5), nota se que, a EAI na forma de aplicação superficial aumentou o valor em relação a incorporada, e o CA quando aplicado na forma incorporada, obteve maior resultado, comparado ao superficial.

Tabela 5. Desdobramento da interação aplicação x corretivos para a saturação por bases (V\%).

Table 5. Deployment of the application x corrective interaction for the base saturation $(\mathrm{V} \%)$.

\begin{tabular}{|c|c|c|}
\hline \multirow{3}{*}{ Corretivos* } & \multicolumn{2}{|c|}{$\begin{array}{c}\text {----- V \% ---------- } \\
\text { Aplicação }\end{array}$} \\
\hline & Incorporada & Superficial \\
\hline & \multicolumn{2}{|c|}{$20-40 \mathrm{~cm}$} \\
\hline EAI & $30,9 \mathrm{bC}$ & $46,0 \mathrm{aA}$ \\
\hline CA & $54,0 \mathrm{aA}$ & $36,4 \mathrm{bA}$ \\
\hline CAC & $40,1 \mathrm{aBC}$ & 36,6 aA \\
\hline EA & $37,7 \mathrm{aBC}$ & $34,0 \mathrm{aAB}$ \\
\hline EFP & $45,5 \mathrm{aAB}$ & $37,0 \mathrm{aA}$ \\
\hline W & 42,6 aABC & $44,1 \mathrm{aA}$ \\
\hline $\mathrm{T}$ & $16,5 \mathrm{aD}$ & $21,0 \mathrm{aB}$ \\
\hline
\end{tabular}

*EAI: escória do aço inox, CA: calcário agrícola, CAC: calcário agrícola calcinado, EA: escória de aciaria, EFP: escória forno de panela, W: wollastonita e T: testemunha. Letras maiúsculas correspondem ás comparações entre os corretivos de acidez do solo dentro do mesmo modo de aplicação. Letras minúsculas correspondem ás comparações entre o modo de aplicação para o mesmo corretivo de acidez do solo pelo teste t de Student $\mathrm{p}>0,05$. 
Para a forma de aplicação incorporada, a aplicação do CA, EFP e a W foram os que mais aumentaram o $\mathrm{V} \%$, não diferindo estatisticamente entre si. O CA elevou a saturação por bases a um valor 3,3 vezes maior que o da testemunha; esta por sua vez, apresentou o menor valor quando comparado ao uso de todos os corretivos. Não houve variação significativa entre os corretivos no sistema de aplicação superficial para os valores de $\mathrm{V} \%$, sendo que, todos corretivos elevaram a saturação por bases do solo em relação à testemunha (Tabela 5).

De acordo com a Tabela 6, verifica se que a forma de aplicação dos corretivos não influenciou nos teores de Ca e $\mathrm{Mg}$, em todas as profundidades avaliadas.

$\mathrm{O}$ cálcio ( $\mathrm{Ca}$ ) apresentou maiores teores quando foi aplicado os corretivos W, EFP, EA e EAI, principalmente nas camadas superficiais. Os calcários não apresentaram diferenças estatísticas entre eles em todas as camadas avaliadas. A EA proporcionou o maior aumento na concentração de $\mathrm{Ca}$ quando comparado ao efeito dos calcários, na camada de $0-5 \mathrm{~cm}$ de profundidade, contudo não diferiu estatisticamente da EAI, EFP e da W.

Já para a camada de $5-10 \mathrm{~cm}$ de profundidade, a W apresentou os maiores valores de $\mathrm{Ca}$, contudo não apresentou diferença significativa em relação a EA e a EAI (Tabela 6).

Nas camadas de $10-20$ e $20-40 \mathrm{~cm}$ de profundidade, observou se que novamente a $\mathrm{W}$ apresentou os maiores teores de $\mathrm{Ca}$ encontrados nestas camadas, contudo, estes efeitos não apresentaram diferenças significativas quando comparados a EAI e a EA; e a T apresentou os menores valores de $\mathrm{Ca}$ registrados em todas as camadas de solo avaliadas.

Para a variável $\mathrm{Mg}$ a aplicação do corretivo CA obteve médias significativas superiores aos demais corretivos em praticamente todas as camadas, isto se deve ao fato de que o calcário agrícola (CA) é o corretivo que contém maior teor de $\mathrm{MgO}$ dentre os demais (Tabela 2). Os teores de $\mathrm{P}$ não foram influenciados pelo método de aplicação dos corretivos até os $40 \mathrm{~cm}$ de profundidade (Tabela 7).

Tabela 6. Valores médios de cálcio (Ca), magnésio (Mg) no solo 8 meses após a reaplicação em superfície de escória do aço inox (EAI), calcário agrícola (CA), calcário agrícola calcinado (CAC), escória de aciaria (EA), escória forno de panela (EFP), wollastonita (W) e testemunha $(\mathrm{T})$.

Table 6. Mean values of calcium $(\mathrm{Ca})$, magnesium $(\mathrm{Mg})$ in the soil 8 months after stainless steel slag (EAI), agricultural calcareous (AC), calcined agricultural limestone (CAC), steel slag (EA), ladle furnace slag (EFP) slag, wollastonite (W) and control (T).

\begin{tabular}{|c|c|c|c|c|c|c|c|c|}
\hline \multirow{2}{*}{ Tratamento } & \multicolumn{4}{|c|}{$\mathrm{Ca}\left(\mathrm{mmol}_{\mathrm{c}} / \mathrm{dm}^{3}\right)$} & \multicolumn{4}{|c|}{$\mathrm{Mg}\left(\mathrm{mmol}_{\mathrm{c}} / \mathrm{dm}^{3}\right)$} \\
\hline & \multicolumn{8}{|c|}{ Camadas $(\mathrm{cm})$} \\
\hline Aplicação (A) & $0-5$ & $5-10$ & $10-20$ & $20-40$ & $0-5$ & $5-10$ & $10-20$ & $20-40$ \\
\hline Incorporada & $42,7 \mathrm{a}$ & $42,2 \mathrm{a}$ & $31,8 \mathrm{a}$ & $26,9 \mathrm{a}$ & $12,7 \mathrm{a}$ & $12,1 \mathrm{a}$ & $10,7 \mathrm{a}$ & $9,5 \mathrm{a}$ \\
\hline Superficial & $52,9 \mathrm{a}$ & $42,3 \mathrm{a}$ & $31,8 \mathrm{a}$ & $26,4 \mathrm{a}$ & $15,1 \mathrm{a}$ & $11,5 \mathrm{a}$ & $10,2 \mathrm{a}$ & $9,4 \mathrm{a}$ \\
\hline $\mathrm{F}$ & $4,4^{\mathrm{ns}}$ & $0,0^{\mathrm{ns}}$ & $0,5^{\text {ns }}$ & $0,1^{\mathrm{ns}}$ & $2,8^{\text {ns }}$ & $0,1^{\mathrm{ns}}$ & $0,1^{\text {ns }}$ & $0,1^{\mathrm{ns}}$ \\
\hline \multicolumn{9}{|l|}{ Corretivos (C) } \\
\hline EAI & $53,7 \mathrm{ab}$ & $49,0 \mathrm{ab}$ & $36,2 \mathrm{ab}$ & $30,4 \mathrm{ab}$ & $15,1 \mathrm{~b}$ & $13,5 \mathrm{~b}$ & $11,6 \mathrm{~b}$ & $10,6 \mathrm{bc}$ \\
\hline $\mathrm{CA}$ & $46,4 \mathrm{~b}$ & $39,9 \mathrm{~cd}$ & $31,8 \mathrm{~b}$ & $24,1 \mathrm{~b}$ & $22,8 \mathrm{a}$ & $19,4 \mathrm{a}$ & $16,9 \mathrm{a}$ & $13,8 \mathrm{a}$ \\
\hline CAC & $45,8 \mathrm{~b}$ & $38,5 \mathrm{~d}$ & $32,0 \mathrm{~b}$ & $27,2 \mathrm{~b}$ & $14,2 \mathrm{~b}$ & $13,5 \mathrm{~b}$ & $12,7 \mathrm{~b}$ & $11,7 \mathrm{ab}$ \\
\hline EA & $63,3 \mathrm{a}$ & $52,0 \mathrm{ab}$ & $36,0 \mathrm{~b}$ & $26,8 \mathrm{~b}$ & $14,6 \mathrm{~b}$ & $9,7 \mathrm{c}$ & $7,0 \mathrm{c}$ & $6,5 \mathrm{~d}$ \\
\hline EFP & $54,7 \mathrm{ab}$ & $48,1 \mathrm{bc}$ & $36,6 \mathrm{ab}$ & $30,4 \mathrm{ab}$ & $13,8 \mathrm{~b}$ & $11,6 \mathrm{bc}$ & $10,3 \mathrm{bc}$ & $10,0 \mathrm{bc}$ \\
\hline W & $58,2 \mathrm{ab}$ & $56,8 \mathrm{a}$ & 44,6 a & $34,5 \mathrm{a}$ & $11,2 \mathrm{~b}$ & $10,0 \mathrm{c}$ & $9,6 \mathrm{bc}$ & $8,1 \mathrm{~cd}$ \\
\hline $\mathrm{T}$ & $12,2 \mathrm{c}$ & $11,4 \mathrm{e}$ & $12,3 \mathrm{c}$ & $12,8 \mathrm{c}$ & $5,6 \mathrm{c}$ & $4,7 \mathrm{~d}$ & $5,0 \mathrm{c}$ & $5,5 \mathrm{~d}$ \\
\hline $\mathrm{F}$ & $9,7^{* *}$ & $26,9^{* *}$ & $11,2^{* *}$ & $9,4^{* *}$ & $9,8^{* *}$ & $23,3^{* *}$ & $11,2^{* *}$ & $8,4^{* *}$ \\
\hline $\mathrm{AxC}$ & $1,4^{\text {ns }}$ & $1,5^{\text {ns }}$ & 0,9 ns & $2,0^{\mathrm{ns}}$ & $0,2^{\text {ns }}$ & $0,814^{\mathrm{ns}}$ & $0,8^{\text {ns }}$ & $0,5^{\text {ns }}$ \\
\hline $\mathrm{CV}_{\text {parcela }}(\%)$ & 38,0 & 42,97 & 33,1 & 61,7 & 38,7 & 54,25 & 59,3 & 66,5 \\
\hline $\mathrm{CV}$ subparcela $(\%)$ & 32,0 & 19,42 & 25,7 & 24,0 & 33,4 & 22,36 & 31,29 & 30,2 \\
\hline
\end{tabular}

Médias seguidas por letras distintas dentro das colunas diferem entre si, pelo teste t de Student ao nível de 5\% (*) e 1\% (**) de probabilidade. ns - não significativo.

Para o teor de P disponível, apenas a camada de $0-5 \mathrm{~cm}$ apresentou diferenças significativas com a aplicação dos diferentes corretivos, na qual a EA foi a que mais contribuiu para o aumento do elemento. Comportamento semelhante já havia sido observado nos primeiros $20 \mathrm{~cm}$ de solo, antes da reaplicação dos corretivos (Tabela 1), onde a EA já apresentava nível médio de $\mathrm{P}$ na forma de aplicação superficial, se comparado com o baixo valor da T.

Nas camadas de $5-10$ e $10-20 \mathrm{~cm}$ de profundidade, os corretivos EAI, EA e EFP foram os que mais influenciaram na disponibilidade do $\mathrm{Si}$; enquanto que a testemunha apresentou $7,6 \mathrm{mg} \mathrm{kg}^{-1}$ e para a camada de $10-20 \mathrm{~cm}$, a EAI, EA e a EFP disponibilizaram 10,2, 9,7 e 9,9 $\mathrm{mg} \mathrm{kg}^{-1}$ de Si no solo, respectivamente.

Um destaque importante para a disponibilidade do $\mathrm{Si}$, a qual apresentou interação significativa entre os modos de aplicação e os corretivos avaliados em duas camadas, a mais superficial $(0-5 \mathrm{~cm})$ e a mais profunda $(20-40 \mathrm{~cm})$ (Tabela 8). Para a camada de $0-5 \mathrm{~cm}$ no sistema de aplicação superficial sem revolvimento ao longo do tempo (Tabela 8), constatou se que os tratamentos com EAI, EA e W apresentaram aumento médio na disponibilidade de Si de $26 \%, 31 \%$ e $23 \%$, respectivamente, em relação à aplicação incorporada. Este acúmulo pode ter sido ocasionado pela deposição dos corretivos ao longo dos anos pelas reaplicações superficiais.

Com relação ao aumento na disponibilidade de Si no solo com a aplicação dos corretivos (Tabela 8), o grupo das escórias (EAI, EA e EFP) e dos calcários (CA e CAC) juntamente com a wollastonita, não apresentaram diferenças entre si, dentro de cada grupo. 
Tabela 7. Valores médios de fósforo $(\mathrm{P})$ e silício $(\mathrm{Si})$ no solo 8 meses após a reaplicação em superfície de escória do aço inox (EAI), calcário agrícola (CA), calcário agrícola calcinado (CAC), escória de aciaria (EA), escória forno de panela (EFP), wollastonita (W) e testemunha (T). Table 7. Mean values of phosphorus $(\mathrm{P})$ and silicon $(\mathrm{Si})$ in the soil 8 months after the application of stainless steel slag (EAI), agricultural limestone (CA), calcined agricultural limestone (CAC), steel slag (EA), ladle furnace slag (EFP) slag, wollastonite (W) and control (T).

\begin{tabular}{|c|c|c|c|c|c|c|c|c|}
\hline \multirow{3}{*}{$\begin{array}{l}\text { Tratamento } \\
\text { Aplicação (A) }\end{array}$} & \multicolumn{4}{|c|}{$\mathrm{P}\left(\mathrm{mg} / \mathrm{dm}^{3}\right)$} & \multicolumn{4}{|c|}{$\mathrm{Si}(\mathrm{mg} / \mathrm{kg})$} \\
\hline & \multicolumn{8}{|c|}{ Camadas $(\mathrm{cm})$} \\
\hline & $0-5$ & $5-10$ & $10-20$ & $20-40$ & $0-5$ & $5-10$ & $10-20$ & $20-40$ \\
\hline Incorporada & $18,5 \mathrm{a}$ & $18,1 \mathrm{a}$ & $11,87 \mathrm{a}$ & $5,1 \mathrm{a}$ & 10,3 & $10,7 \mathrm{a}$ & $9,1 \mathrm{a}$ & 8,2 \\
\hline Superficial & $22,0 \mathrm{a}$ & $22,2 \mathrm{a}$ & $11,05 \mathrm{a}$ & $5,7 \mathrm{a}$ & 12,6 & $11,0 \mathrm{a}$ & $9,1 \mathrm{a}$ & 8,6 \\
\hline $\mathrm{F}$ & $3,4^{\text {ns }}$ & $1,8^{\mathrm{ns}}$ & $0,2^{\mathrm{ns}}$ & $0,2^{\mathrm{ns}}$ & $11,1^{*}$ & $0,2^{\mathrm{ns}}$ & $0,1^{\mathrm{ns}}$ & $1,9^{\text {ns }}$ \\
\hline \multicolumn{9}{|l|}{ Corretivos $(\mathrm{C})$} \\
\hline EAI & $18,2 \mathrm{~b}$ & $22,8 \mathrm{a}$ & $10,0 \mathrm{a}$ & $6,1 \mathrm{a}$ & 14,8 & $13,9 \mathrm{a}$ & $10,2 \mathrm{a}$ & 9,9 \\
\hline $\mathrm{CA}$ & $15,0 \mathrm{~b}$ & $18,7 \mathrm{a}$ & $11,2 \mathrm{a}$ & $5,0 \mathrm{a}$ & 10,5 & $9,9 \mathrm{c}$ & $8,6 \mathrm{ab}$ & 7,5 \\
\hline $\mathrm{CAC}$ & $16,7 \mathrm{~b}$ & $16,2 \mathrm{a}$ & $10,3 \mathrm{a}$ & $4,8 \mathrm{a}$ & 9,3 & $9,5 \mathrm{c}$ & $8,7 \mathrm{ab}$ & 7,7 \\
\hline EA & $37,8 \mathrm{a}$ & $29,7 \mathrm{a}$ & $17,4 \mathrm{a}$ & $7,0 \mathrm{a}$ & 14,5 & $12,2 \mathrm{~b}$ & $9,7 \mathrm{a}$ & 9,0 \\
\hline EFP & $17,2 \mathrm{~b}$ & $18,0 \mathrm{a}$ & $8,3 \mathrm{a}$ & $5,4 \mathrm{a}$ & 13,9 & $12,6 \mathrm{ab}$ & $9,9 \mathrm{a}$ & 8,9 \\
\hline W & $18,1 \mathrm{~b}$ & $15,5 \mathrm{a}$ & $12,1 \mathrm{a}$ & $5,1 \mathrm{a}$ & 9,8 & $10,3 \mathrm{c}$ & $9,2 \mathrm{ab}$ & 8,3 \\
\hline $\mathrm{T}$ & $18,8 \mathrm{~b}$ & $20,2 \mathrm{a}$ & $11,2 \mathrm{a}$ & $4,4 \mathrm{a}$ & 7,3 & $7,6 \mathrm{~d}$ & $7,5 \mathrm{~b}$ & 7,4 \\
\hline $\mathrm{F}$ & $12,6^{* *}$ & $1,9^{\mathrm{ns}}$ & $2,2^{\mathrm{ns}}$ & $0,7^{\mathrm{ns}}$ & $22,5^{* *}$ & $17,5^{* *}$ & $2,4^{*}$ & $9,2^{* *}$ \\
\hline $\mathrm{AxC}$ & $0,7^{\mathrm{ns}}$ & $1,1^{\mathrm{ns}}$ & $0,2^{\mathrm{ns}}$ & $0,6^{\mathrm{ns}}$ & $2,04^{*}$ & $1,5^{\mathrm{ns}}$ & $0,8^{\text {ns }}$ & $4,1^{* *}$ \\
\hline $\mathrm{CV}$ parcela $(\%)$ & 35,5 & 56,9 & 60,0 & 73,3 & 22,3 & 20,32 & 9,5 & 14,7 \\
\hline CV subparcela $(\%)$ & 30,7 & 49,8 & 47,2 & 53,44 & 15,4 & 13,49 & 18,5 & 10,1 \\
\hline
\end{tabular}

Médias seguidas por letras distintas dentro das colunas diferem entre si, pelo teste t de Student ao nível de $5 \%(*)$ e $1 \%$ (**) de probabilidade. ns - não significativo.

Tabela 8. Desdobramento da interação aplicação x corretivos para o teor de silício no solo (Si).

Table 8. Deployment of the interaction x corrective to the silicon content in the soil $(\mathrm{Si})$

\begin{tabular}{lcccc}
\hline & \multicolumn{3}{c}{ Aplicação } \\
\cline { 2 - 5 } Corretivos* & Incorporada & Superficial & Incorporada & Superficial \\
\cline { 2 - 5 } & & $0-5 \mathrm{~cm}$ & & $20-40 \mathrm{~cm}$ \\
\hline EAI & $12,6 \mathrm{bA}$ & $17,1 \mathrm{aAB}$ & $8,7 \mathrm{bAB}$ & $11,1 \mathrm{aA}$ \\
CA & $10,1 \mathrm{aBC}$ & $11,0 \mathrm{aC}$ & $7,9 \mathrm{aBC}$ & $7,2 \mathrm{aD}$ \\
CAC & $9,0 \mathrm{aCD}$ & $9,7 \mathrm{aCD}$ & $7,1 \mathrm{bC}$ & $8,5 \mathrm{aBC}$ \\
EA & $11,9 \mathrm{bAB}$ & $17,2 \mathrm{aA}$ & $8,8 \mathrm{aAB}$ & $9,3 \mathrm{aB}$ \\
EFP & $13,1 \mathrm{aA}$ & $14,6 \mathrm{aB}$ & $9,6 \mathrm{aA}$ & $8,3 \mathrm{bBCD}$ \\
W & $8,5 \mathrm{bCD}$ & $11,1 \mathrm{aC}$ & $8,3 \mathrm{aAB}$ & $8,3 \mathrm{aBCD}$ \\
T & $7,0 \mathrm{aD}$ & $7,6 \mathrm{aD}$ & $7,0 \mathrm{aC}$ & $7,8 \mathrm{aCD}$ \\
\hline
\end{tabular}

*EAI: escória do aço inox, CA: calcário agrícola, CAC: calcário agrícola calcinado, EA: escória de aciaria, EFP: escória forno de panela, W: wollastonita e T: testemunha. Letras maiúsculas correspondem ás comparações entre os corretivos de acidez do solo dentro do mesmo modo de aplicação. Letras minúsculas correspondem ás comparações entre o modo de aplicação para o mesmo corretivo de acidez do solo pelo teste $t$ de Student $\mathrm{p}>0,05$.

A W apresentou resultado similar a testemunha, embora sendo um silicato não ocasionou incremento nos níveis de Si no solo. Esses resultados corroboram com os resultados encontrados por Deus (2014), confirmando que após 6 anos da aplicação inicial dos corretivos EAI, EA e EFP realmente mostram ser mais efetivos na disponibilização do Si para o solo. E prova também que, embora a $\mathrm{W}$ possua Si em sua composição, tem baixa efetividade no incremento deste nutriente no solo.

$\mathrm{Na}$ camada de $20-40 \mathrm{~cm}$ de profundidade do solo, com relação aos corretivos em função do modo de aplicação, observou se que, a EAI e o CAC apresentaram diferenças significativas no aumento da disponibilidade de Si no solo quando aplicado superficialmente $22 \%$ e $16 \%$, respectivamente; a EFP obteve aumento significativo na disponibilidade quando foi incorporada ao solo (13\%) (Tabela 8).

Já quanto a ação da forma de aplicação sobre os corretivos, observou se que, para a forma de aplicação incorporada, a EFP apresentou o maior aumento significativo na disponibilidade de $\mathrm{Si}$ em relação a $\mathrm{T}$, com incremento de $27 \%$, contudo, a EFP não se diferiu estatisticamente da EAI, EA e da W. Quanto a forma de aplicação superficial, foi constatado que, a EAI foi o corretivo que mais aumentou a disponibilidade de Si no solo, com acréscimo de 30\% (Tabela 8).

A forma de aplicação não interferiu nos componentes de produção da soja: número de plantas por hectare, número de vagens por planta, número de grãos por vagem, massa de 100 grãos e produtividade de grãos (Tabela 9).

Os resultados de produtividade com a aplicação dos corretivos mostram se satisfatórios (Tabela 9), pois todos aumentaram a produtividade de grãos da soja em relação a $T$, com destaque para a EA, EAI e a W, os quais não diferiam estatisticamente entre si. O CA e CAC apresentaram valores intermediários de produtividade de grãos, e não diferiram estatisticamente da EAI nem da W. A testemunha foi o tratamento que obteve a menor produtividade média de grãos de soja.

Com o uso da escória de aciaria na correção da acidez do solo houve um incremento de produtividade média de 54\% a mais que o obtido no tratamento sem correção do solo (T). E quando comparado o efeito da escória de aciaria com a produtividade média dos calcários, observou se que a superioridade da aciaria foi de $22 \%$ em relação à média produtiva dos calcários. 
Tabela 9. Componentes da produção e produtividade média de grãos de soja após a reaplicação em superfície de escória do aço inox (EAI), calcário agrícola (CA), calcário agrícola calcinado (CAC), escória de aciaria (EA), escória forno de panela (EFP), wollastonita (W) e testemunha $(\mathrm{T})$.

Table 9. Components of the production and average yield of soybeans after the application of stainless steel slag (EAI), agricultural limestone (CA), calcined agricultural limestone (CAC), steel slag (EA), ladle furnace slag (EFP), wollastonite (W) and control (T).

\begin{tabular}{|c|c|c|c|c|c|}
\hline Tratamentos & $\mathrm{N}^{\mathrm{o}}$ de Plantas ha ${ }^{-1}$ & $\begin{array}{c}\mathrm{N}^{\circ} \text { de Vagens por } \\
\text { Planta }\end{array}$ & $\begin{array}{c}\mathrm{N}^{\circ} \text { de grãos por } \\
\text { vagem }\end{array}$ & $\begin{array}{l}\text { Massa de } 100 \\
\text { grãos }(\mathrm{g})\end{array}$ & $\begin{array}{c}\text { Produtividade de grãos } \\
\left(\mathrm{Mg} \mathrm{ha}^{-1}\right)\end{array}$ \\
\hline \multicolumn{6}{|l|}{ Aplicação (A) } \\
\hline Incorporada & $432.738 a$ & $32 \mathrm{a}$ & $2 \mathrm{a}$ & $27,23 \mathrm{a}$ & $5,38 \mathrm{a}$ \\
\hline Superficial & $410.119 \mathrm{a}$ & $31 \mathrm{a}$ & $2 \mathrm{a}$ & $27,14 \mathrm{a}$ & $5,33 \mathrm{a}$ \\
\hline $\mathrm{F}$ & $0,3^{\text {ns }}$ & $0,1^{\text {ns }}$ & $1,0^{\mathrm{ns}}$ & $0,1^{\mathrm{ns}}$ & $0,1^{\mathrm{ns}}$ \\
\hline \multicolumn{6}{|l|}{ Corretivos (C) } \\
\hline EAI & $361.111 \mathrm{a}$ & $37 \mathrm{a}$ & $2 a$ & $28,03 \mathrm{a}$ & $6,11 \mathrm{ab}$ \\
\hline $\mathrm{CA}$ & $406.249 \mathrm{a}$ & $24 \mathrm{a}$ & $2 \mathrm{a}$ & $28,06 \mathrm{a}$ & $5,32 \mathrm{bc}$ \\
\hline CAC & $393.749 \mathrm{a}$ & $34 \mathrm{a}$ & $2 \mathrm{a}$ & $27,81 \mathrm{a}$ & $5,62 \mathrm{bc}$ \\
\hline EA & $439.583 \mathrm{a}$ & $34 \mathrm{a}$ & $2 \mathrm{a}$ & $27,01 \mathrm{a}$ & $7,02 \mathrm{a}$ \\
\hline EFP & $441.666 \mathrm{a}$ & $34 \mathrm{a}$ & $2 \mathrm{a}$ & $24,36 \mathrm{a}$ & $4,34 \mathrm{~cd}$ \\
\hline W & $465.277 \mathrm{a}$ & $32 \mathrm{a}$ & $2 \mathrm{a}$ & $27,82 \mathrm{a}$ & $5,87 \mathrm{ab}$ \\
\hline $\mathrm{T}$ & $442.361 \mathrm{a}$ & $28 \mathrm{a}$ & $2 \mathrm{a}$ & $27,16 \mathrm{a}$ & $3,25 \mathrm{~d}$ \\
\hline $\mathrm{F}$ & $1,8^{\mathrm{ns}}$ & $2,3^{\text {ns }}$ & $1,6^{\mathrm{ns}}$ & $1,5^{\mathrm{ns}}$ & $7,1^{* *}$ \\
\hline $\mathrm{AxC}$ & $1,7^{\mathrm{ns}}$ & $0,3^{\text {ns }}$ & $0,8^{\text {ns }}$ & $0,8^{\text {ns }}$ & $1,5^{\mathrm{ns}}$ \\
\hline CV parcela $(\%)$ & 37,6 & 59,4 & 19,4 & 15,1 & 49,6 \\
\hline $\mathrm{CV}$ subparcela $(\%)$ & 18,0 & 26,4 & 16,1 & 11,0 & 24,3 \\
\hline
\end{tabular}

Médias seguidas por letras distintas dentro das colunas diferem entre si, pelo teste t de Student ao nível de $5 \%(*)$ e $1 \%(* *)$ de probabilidade. ns - não significativo.

A produção de matéria seca (MS) da soja não apresentou influência significativa para o sistema de aplicação, nem para os corretivos aplicados (Tabela 10). Para o acúmulo médio de silício nas plantas de soja, não foram observados incrementos distintos significativamente entre os modos de aplicação, nem para os diferentes tipos de corretivos avaliados (Tabela 10). Com isso, evidencia se que o uso dos corretivos de acidez do solo, não aumentou significativamente o acúmulo de Si nas plantas de soja.

Tabela 10. Produção média de matéria seca (MS) e acúmulo médio de silício por planta na soja em função da reaplicação em superfície de escória do aço inox (EAI), calcário agrícola (CA), calcário agrícola calcinado (CAC), escória de aciaria (EA), escória forno de panela (EFP), wollastonita (W) e testemunha (T).

Table 10. Average dry matter (DM) production and average silicon accumulation per plant in soybean as a function of the reapplication on the surface of stainless steel slag (EAI), agricultural limestone (CA), calcined agricultural limestone (CAC), steel slag (EA), pan boiler slag (EFP), wollastonite (W) and control (T).

\begin{tabular}{lcc}
\hline Tratamento & MS soja & Acúmulo de Si $\left(\mathrm{g} \mathrm{kg}^{-1}\right)$ \\
\hline Aplicação $(\mathrm{A})$ & & \\
\hline Incorporado & $29,03 \mathrm{a}$ & $31,93 \mathrm{a}$ \\
Superficial & $27,20 \mathrm{a}$ & $32,61 \mathrm{a}$ \\
\hline $\mathrm{F}$ & $0,7^{\mathrm{ns}}$ & $0,4^{\mathrm{ns}}$ \\
\hline Corretivos $(\mathrm{C})$ & & \\
\hline EAI & $29,28 \mathrm{a}$ & $38,30 \mathrm{a}$ \\
$\mathrm{CA}$ & $27,80 \mathrm{a}$ & $27,64 \mathrm{a}$ \\
$\mathrm{CAC}$ & $25,70 \mathrm{a}$ & $25,72 \mathrm{a}$ \\
EA & $35,43 \mathrm{a}$ & $32,34 \mathrm{a}$ \\
EFP & $24,95 \mathrm{a}$ & $33,94 \mathrm{a}$ \\
$\mathrm{T}$ & $22,25 \mathrm{a}$ & $30,97 \mathrm{a}$ \\
W & $31,45 \mathrm{a}$ & $37,00 \mathrm{a}$ \\
\hline F & $2,1^{\mathrm{ns}}$ & $0,9 \mathrm{~ns}$ \\
\hline AxC & $0,8^{\mathrm{ns}}$ & $0,8^{\mathrm{ns}}$ \\
$\mathrm{CV}$ & 38,0 \\
$\mathrm{CV}$ & 28,4 & 42,1 \\
\hline
\end{tabular}

Médias seguidas por letras distintas dentro das colunas diferem entre si, pelo teste $\mathrm{t}$ de Student ao nível de $5 \%\left(^{*}\right)$ e $1 \%(* *)$ de probabilidade. ns - não significativo.

\section{DISCUSSÃO}

A reação de neutralização da acidez do solo pela escória, ocorre quando se forma o ácido monossilícico $\left(\mathrm{H}_{4} \mathrm{SiO}_{4}\right)$, proveniente da reação entre $\mathrm{o}^{+}$da solução do solo com o $\mathrm{SiO}_{3}$ oriundo da dissociação do $\mathrm{CaSiO}_{3}$ e do $\mathrm{MgSiO}_{3}$ em Ca, $\mathrm{Mg}$ e $\mathrm{SiO}^{3}$, reduzindo assim sua acidez (PREZOTTI; MARTINS, 2012).

Estudos realizados por Prado; Fernandes (2003) demonstraram que a escória, quando aplicada ao solo, aumenta o $\mathrm{pH}$ e reduz o $\mathrm{H}+\mathrm{Al}$.

Estudando o efeito da aplicação de silicatos e carbonatos Nolla et al. (2013), constatou que os silicatos e os carbonatos foram eficazes na neutralização do $\mathrm{Al}^{3+}$, beneficiando assim o desenvolvimento das culturas.

Para a calagem superficial ter efeito nas camadas subsuperficiais, ela depende de condições favoráveis para a formação e migração de $\mathrm{Ca}\left(\mathrm{HCO}_{3}\right)_{2}$ e $\mathrm{Mg}\left(\mathrm{HCO}_{3}\right)_{2}$ (OLIVEIRA; PAVAN, 1996; CAIRES et al., 2005); através do deslocamento vertical de partículas finas do corretivo, juntamente com o movimento de água descendente no perfil do solo (AMARAL et al., 2004).

Segundo Prezotti; Martins (2012), a aplicação de escória siderúrgica aumentou a saturação de bases aos três e seis meses após a aplicação do corretivo. Assim como observado por Rodrighero et al. (2015), quando a aplicação superficial de calcário, após 12 meses, proporcionou redução da acidez na camada de $0-5 \mathrm{~cm}$ e, em menor grau, na de $5-10 \mathrm{~cm}$ e consequentemente aumento da saturação de base.

Os resultados obtidos com a aplicação dos silicatos sobre a elevação dos teores de Ca no solo, foram superiores a influência que os carbonatos obtiveram, mostrando se contrários aos resultados mencionados por Nolla et al. (2013), onde observou uma redução eficiente na acidez do 
solo, contudo, o silicato disponibilizou menos Ca em solução em relação ao carbonato.

A disponibilização de $\mathrm{Mg}$ pelo CA foi superior aos demais tratamentos devido a maior concentração de $\mathrm{Mg}$ presente neste corretivo. Esse fato é discutido por Nolla et al. (2013) que relatou que mesmo usando uma fonte pobre em $\mathrm{Mg}$ e realizando uma adubação com magnésio, o carbonato se saiu melhor na disponibilidade de $\mathrm{Mg}$ no solo, apresentando maior eficiência quando comparado ao silicato de cálcio.

Resultados semelhantes com aumento na disponibilidade de P com a aplicação de escória de aciaria foram observados por Corrêa et al. (2008). A maior disponibilidade de P para a EA, pode estar relacionada com a natureza química dos corretivos, como pode se observar nos valores de $\mathrm{P}$ na composição da EA (11,0 g. $\left.\mathrm{kg}^{-1}\right)$ apresentados por Deus (2013), na caracterização química destes materiais.

Segundo o relato de Corrêa et al. (2008), onde obteve resultado semelhante de aumento na disponibilidade de $\mathrm{P}$, em função da aplicação da EA, a maior disponibilidade de $\mathrm{P}$ pode estar associada também pela competição do ânion $\mathrm{SiO}_{4}^{-4}$ pelos sítios de adsorção do $\mathrm{P}$, fazendo com que este nutriente esteja mais disponível (PREZOTTTI; MARTINS, 2012; PRADO; FERNANDES, 2003).

A disponibilidade de $\mathrm{Si}$ pelos silicatos mostrou se eficiente, exceto para a W; resultados semelhantes foram encontrados por Deus (2014), mostrando a eficiência da EAI, EA e EFP em disponibilizar o Si no perfil do solo.

Esse aumento de produtividade de grãos da cultura da soja, possivelmente foi provocado pela maior quantidade de Ca encontrados nos tratamentos com aplicação de EAI e W em todas as camadas $(0-40 \mathrm{~cm})$ de solo e para a EA nas camadas de $0-10 \mathrm{~cm}$ de profundidade (Tabela 6$)$. E para a maior quantidade de $\mathrm{P}$ e Si na camada de $0-5 \mathrm{~cm}$ resultante da aplicação de EAI e EA (Tabelas 7). A maior produtividade das plantas de soja pode estar associada também a quantidade de $\mathrm{P}$ fornecido nas adubações de semeadura e da ressemeadura da cultura. Estas maiores quantidades de nutrientes nas camadas superficiais provavelmente foram fundamentais para as maiores produtividades, uma vez que, na camada de $0-20 \mathrm{~cm}$ do solo é onde se localiza a maior concentração do sistema radicular das plantas.

Avaliando a influência na nutrição e produtividade de tubérculos de batata, em função da aplicação do silicato de cálcio e magnésio e calcário na correção da acidez do solo, Pulz et al. (2008) relatou que os silicatos se sobressaíram em relação ao calcário, aumentando a disponibilidade de P e Si no solo, sendo estes absorvidos em maior quantidade, melhorando a arquitetura e aumentando assim a produtividade da cultura. Desta forma, as escórias de siderurgia mostram ser uma ótima alternativa para o incremento da produtividade nos cultivos em SPD, principalmente em regiões com maior disponibilidade desses corretivos, e ainda disponibilizando Si para o solo.

\section{CONCLUSÕES}

Os corretivos da acidez do solo avaliados, influenciaram na correção da acidez até a profundidade $40 \mathrm{~cm}$. A wollastonita, escória do aço inox e o calcário agrícola apresentaram maior influência na neutralização da acidez do solo até $10 \mathrm{~cm}$. Os melhores resultados com a aplicação dos silicatos no solo foram o aumento nos teores de P, K, Ca e $\mathrm{Si}$, e nos valores de $\mathrm{SB}, \mathrm{V} \%$ e $\mathrm{pH}$.
A escória de aciaria, escória do aço inox e a wollastonita, destacaram se por proporcionaram as maiores produtividades de grãos para a cultura da soja.

\section{REFERÊNCIAS}

ALCARDE, J. C. Corretivos da acidez dos solos: características e interpretações técnicas. São Paulo: ANDA, 1992. (Boletim Técnico, 6).

AMARAL, A. S.; ANGHINONI, I.; BERTOL, I.; HINRICHS, R. Movimentação de partículas de calcário no perfil de um Cambissolo em plantio direto. Revista Brasileira de Ciência do Solo, Viçosa, v. 28, p. 359-367, 2004. DOI: http://dx.doi.org/10.1590/S010006832004000200014

CAIRES, E. F.; KUSMAN, M. T.; BARTH, G.; GARBUIO, F. J.; PADILHA, J. M. Alterações químicas do solo e resposta do milho à calagem e aplicação de gesso. Revista Brasileira de Ciência do Solo, Viçosa, v. 28, n. 1, p. 125136, 2004. DOI: http://dx.doi.org/10.1590/S010006832004000100013

CAIRES, E. F.; ALLEONI, L. R. F.; CAMBRI, M. A.; BARTH, G. Surface application of lime for crop grain production under a no-till system. Agronomy Journal, Madison, v. 97, p.791-798, 2005. DOI: https://dx.doi.org/10.2134/agronj2004.0207

CASTRO, G. S. A.; CRUSCIOL, C. A. C. Effects of superficial liming and silicate application on soil fertility and crop yield under rotation. Geoderma, Amsterdam, v.195-96, p.234-242, 2013. DOI: https://dx.doi.org/10.1016/j.geoderma.2012.12.006

CORRÊA, J. C. BÜLL, L. T., CRUSCIOL, C. A. C., TECCHIO, M. A. Correção da acidez e mobilidade de íons em Latossolo com aplicação superficial de escória, lama cal, lodos de esgoto e calcário. Pesquisa Agropecuária Brasileira, Brasília, v. 42, n. 9, p. $1307-$ 1317, 2007. DOI: http://dx.doi.org/10.1590/S0100204X2007000900013

CORRÊA, J. C.; BÜlL, L. T.; CRUSCIOL, C. A. C.; FERNANDES, D. M.; PERES, M. G. M. Aplicação superficial de diferentes fontes de corretivos no crescimento radicular e produtividade da aveia preta. Revista Brasileira de Ciência do Solo, Viçosa, v. 32, n. 4, p. 1583-1590, 2008. DOI: http:/ /dx.doi.org/10.1590/S0100-06832008000400022

CUNHA, A. R.; MARTINS, D. Classificação climática para os municípios de Botucatu e São Manuel, SP. Irriga, Botucatu, v.14, n.1, p.1-11, 2009. DOI: https://dx.doi.org/10.15809/irriga.2009v14n1p1-11

DEUS, A. C. F.; BÜLL, L. T. Eficiência de escórias de siderurgia na cultura do feijoeiro em sistema de semeadura direta. Ciência Rural, Viçosa, v. 43, n. 10, p. 1783-1789, 2013.2 DOI: http://dx.doi.org/10.1590/S0103-84782013005000120

DEUS, A. C. F. Aplicação de corretivos de acidez do solo na implantação do sistema plantio direto. 2014. $130 \mathrm{f}$. Tese (Doutorado) -Universidade Estadual Paulista Júlio De Mesquita Filho, Botucatu, 2014.

EMBRAPA_EMPRESA BRASILEIRA DE PESQUISA AGROPECUÁRIA. Sistema Brasileiro de Classificação de Solos. 3. ed. Brasília: Embrapa Solos, 2013. 353 p. 
KORNDÖRFER, G. H., PEREIRA, H. S., NOLLA, A. Análise de silício no solo, planta e fertilizante. Uberlândia: GPSi, 2004. 50 p. (Boletim Técnico, 02).

LOMBARDI NETO, F.; DRUGOWICH, M. I. Manual técnico de manejo e conservação de solo e água. Campinas: CATI, 1994. v. 2. 168 p.

MAPA_MINISTÉRIO DA AGRICULTURA, PECUÁRIA E ABASTECIMENTO. Manual de métodos analíticos oficiais para fertilizantes e corretivos. 2014. 220 p.

NOLLA, A. Correção da acidez do solo com silicatos. In: SIMPÓSIO SOBRE SILÍCIO NA AGRICULTURA. 3., Uberlândia, 2004. Palestras... Uberlândia: GPSi/ICIAG/UFU, 2004. CD-ROM

NOLLA, A. KORNDÖRFER; PRODUÇÃO DE CANADEAÇÚCAR, G. H. Correção da acidez de um Neosssolo submetido à aplicação de carbonato e silicato de cálcio. Journal of Agronomic Sciences, Umuarama, v.2, n.1, p.113-120, 2013.

OLIVEIRA, E. L.; PAVAN, M. A. Control of soil acidity in no-tillage system for soybean production. Soil and Tillage Research, Amsterdan, v. 38, n. 1-2, p. 47-57, 1996. DOI: https://dx.doi.org/10.1016/01671987(96)01021-5

PRADO, R. M.; FERNANDES, F. M. Efeito residual da escória de siderurgia como corretivo da acidez do solo na soqueira de cana-de-açúcar. Revista Brasileira de
Ciência do Solo, Viçosa, v.27, p.287-286, 2003. DOI: http:/ /dx.doi.org/10.1590/S0100-06832003000200009

PREZOTTI, L. C.; MARTINS, A. G. Efeito da escória de siderurgia na química do solo e na absorção de nutrientes e metais pesados pela cana-de-açúcar. Revista Ceres, Viçosa, v. 59, n.4, p. 530-536, 2012. DOI: http://dx.doi.org/10.1590/S0034-737X2012000400014

PULZ, A. L.; CRUSCIOL, C. A. C.; LEMOS, L. B.; SORATTO, R. P. Influência de silicato e calcário na nutrição, produtividade e qualidade de batata sob deficiência hídrica. Revista Brasileira de Ciência do Solo,Viçosa, v.32, n. 4, p.1651-1659, 2008. DOI: http://dx.doi.org/10.1590/S0100-06832008000400030

RAIJ, B. van; ANDRADE, J. C.; CANTARELLA, H.; QUAGGIO, J. A. Análise Química para avaliação da fertilidade de solos tropicais. Campinas: Instituto Agronômico, 2001.285p.

RODRIGHERO, M. B.; BARTH, G.; CAIRES, E. F. Aplicação superficial de calcário com diferentes teores de magnésio e granulometrias em sistema plantio direto. Revista Brasileira Ciências do Solo, Viçosa, v. 39, n. 6, p.1723-1736, 2015.2 DOI: http://dx.doi.org/10.1590/01000683rbcs20150036

TAYLOR, G. J. The physiologyofaluminumphytotoxicity. In: SIEGAL, H.; SIEGAL, A. (Ed.) MetalsIons in Biological Systems. New York: Marcel Dekker, 1988. p. 123-163. 